\title{
Cutaneous melanoma. Lymph node staging based on current evidence
}

\author{
José Francisco Gallegos-Hernández \\ Instituto Mexicano del Seguro Social, Centro Médico Nacional Siglo XXI, Oncology Hospital, Ciudad de México, Mexico
}

\begin{abstract}
Most patients with cutaneous melanoma present with clinical stage 1 at diagnosis, i.e., with no evidence of lymph node or systemic metastases. However, since this is a type of neoplasm with high affinity to lymphatic tissue, between 30 and $60 \%$ of patients are estimated to have occult metastases on the lymph nodes of the area that drains the primary tumor site at the moment of diagnosis. This possibility depends on several histologic factors, especially thickness of the neoplasm. Historically, in order to reduce the rate of regional recurrence, lymphadenectomy was an essential part of cutaneous melanoma treatment, which has associated morbidity. In the decade of 1990, Morton et al. reported that lymph is initially received by a single lymph node in the lymphatic basin and that its histological status predicts the status of the others and that, therefore, in patients with sentinel lymph node free of metastases lymphadenectomy is not necessary, which reduces morbidity. In the present manuscript, indications, contraindications and requirements for sentinel lymph node identification are described, as well as its current value in cutaneous melanoma diagnostic and therapeutic process.
\end{abstract}

KEY WORDS: Cutaneous melanoma. Sentinel lymph node. Lymphadenectomy. Lymph nodes.

About $85 \%$ of patients with cutaneous melanoma diagnosis are estimated to present with local disease, without evidence of lymph node or systemic metastases; however, the risk of occult lymph node metastases depends on the thickness of the melanoma (Breslow's depth), the presence of ulceration and the mitotic index. In patients with Breslow $<1 \mathrm{~mm}$, prognosis is excellent and the rate of metastasis is low, not so in those who have greater thickness, ulceration or mitotic index $>1$ mitosis per $\mathrm{mm}^{2}$; in this group of patients, identification of occult lymph node metastases is crucial for treatment.

Traditionally, and before the 1990s, lymph node staging was surgically performed, with lymphadenectomy of the area at risk of metastasis (inguinal, axillary or cervical dissection, according to the melanoma anatomical site) being performed; however, these procedures, which are not free of complications, have been replaced by sentinel lymph node biopsy, a procedure known as lymphatic mapping with sentinel lymph node biopsy (SLNB), ${ }^{1}$ which was developed, described and published by Donald Morton and Alistair Cochran in 1992. ${ }^{2}$ This technique is based on the initial works of Cabañas in patients with penile cancer and is the current standard procedure for the staging of skin melanoma patients with Breslow $>1 \mathrm{~mm}$ and without palpable lymph nodes in the area of lymphatic drainage. ${ }^{3}$

Lymphatic mapping with SLNB is one of the most important advances in oncological surgery in the last decades; not only the dilemma of deciding between elective and therapeutic dissection in melanomas with intermediate thickness, but its importance has been such that the technique is fundamental for lymph node staging of many other neoplasms, mainly breast cancer, non-melanoma skin cancer and head and neck mucosal cancer. ${ }^{3}$

It is a minimally invasive technique that in spite of not being without complications, these are less common and serious than those of patients undergoing lymph node dissection.

Lymphatic mapping with SLNB allows, with a lower rate of complications, to classify patients with stage I
Correspondence:

José Francisco Gallegos-Hernández

E-mail: gal61@prodigy.net.mx
Date of reception: 15-01-2018

Date of acceptance: 30-01-2018

DOI: 10.24875/GMM.M19000214
Gac Med Mex. 2018;154:612-614

Contents available at PubMed www.gacetamedicademexico.com 
and II skin melanoma by risk based on the presence or absence of subclinical lymph node metastases (previously unidentified).

The technique must meet at least five requirements:

1. Taking care of the technical aspects that allow a high rate of sentinel lymph node identification and a minimal rate of false-negative results.

2. A low rate of complications.

3. Adequate histological assessment, including immunohistochemistry.

4. Carefully interpreting the sensitivity of the study.

5. Proper selection of patients who are candidate for it, including the diagnostic process.

The technique whereby lymphatic mapping with SLNB is carried out is essential in order to ensure proper identification of the first draining lymph node; without a doubt, the combined technique (dye and radiocolloid) is the standard procedure and should be carried out during the resection of the primary tumor. If the pigmented lesion has been previously excised, the biopsy should not be wide and reconstructions of the area should not be made (flaps or grafts).

The purposes of adequate biopsy and mapping are to obtain a high identification rate and a minimal false-negative results.

Currently, the accepted rate of sentinel lymph node identification is $99.4 \%$ and the proportion of false-negatives depends on primary tumor Breslow's depth: it is $4.8 \%$ in patients with intermediate melanoma and $10.5 \%$ in patients with thick melanoma, based on the results obtained in the MSLT-1 study by Morton. ${ }^{4}$

For adequate identification of the true sentinel lymph node, the nodular area of first drainage should be free of palpable lymph nodes; it is advisable to obtain an ultrasound not showing lymph nodes with suspected metastasis, and if this is the case, ultrasound-guided aspiration biopsy should be carried out; positivity for metastases in this biopsy rules out the patient for the technique and traditional lymphadenectomy should be performed.

Biopsies with wide margins, grafts or rotation of any kind flaps in the area of the primary tumor should be avoided. The use of perilesional rhenium colloid on the eve of the intervention or up to two hours before the procedure is the ideal nuclear medicine method to carry out the "mapping".

Preoperative scintigraphy should be performed (two-dimensional image), which allows anatomical localization (three-dimensional identification with single photon emission computed tomography/computed tomography can be carried out, especially in sites of complex drainage such as head and neck) and the sentinel node drainage or location site should be marked on the skin. Patent blue $\mathrm{V}$ is the ideal dye; it is injected in a perilesional form at least 10 minutes prior to the procedure; the lymph node is identified by its blue staining and its radioactivity and should be located following the stained afferent duct; once excised, intraoperative palpation should be performed (through the incision) to rule out the presence of lymph nodes suspicious to the touch that have not received the colloid or the dye (verbal communication from Dr. Merrick Ross).

The steps above allow obtaining the highest possible identification rate and minimizing the rate of false-negatives and, together with an adequate histopathological examination, it allows to identify submicroscopic metastases; avoiding examination by freezing and waiting for definitive evaluation is recommended. Negative sentinel lymph nodes with standard examination (staining with hematoxylin and eosin) should be studied with immunohistochemical stains (HMB-45 or Melan-A).

The probability of having a metastatic sentinel lymph node ranges from 5 to $40 \%$ and it mainly depends on Breslow's depth. Although other factors have been associated, they are not yet clearly defined and are the subject of controversy, including Clark's level, the anatomical site (higher risk in head and neck melanomas), regression of the primary tumor, ulceration (probably the second most important factor after tumor thickness), lymphovascular invasion, the mitotic index (in those with Breslow lower than $1 \mathrm{~mm}$ ) and age (more often it is metastatic in subjects younger than 20 and rarely it contains metastases in people older than 80 years). ${ }^{5}$

Both retrospectively and recently prospectively, ${ }^{4}$ the prognostic value of sentinel lymph node has been confirmed, in such a way that its histological status is an independent factor, as well as tumor thickness. Thus, in patients with melanomas with Breslow of $1.2 \mathrm{~mm}$ or more, survival is lower and disease-free interval is shorter in patients with metastatic sentinel lymph node, when compared with those with sentinel lymph node negative to metastasis.

On the other hand, sentinel lymph node therapeutic value is poor, i.e., the possibility for SLNB in patients with metastatic sentinel lymph node to prevent neoplastic progression is questionable. In the MSLT-1 study, survival is not better in patients undergoing SLNB in comparison with those who remained under observation, and the disease-free interval is only better by $7 \%$ in intermediate melanomas and $10 \%$ in 
thick melanomas, that is, patients who undergo SLNB have lower relapse rate in the mapped area in comparison with those who remained under observation.

In patients with sentinel lymph node positive for metastasis, the number, as well as the size and site of metastasis are prognostic factors for recurrence and survival.

Patients with thin melanomas, Breslow $<1 \mathrm{~mm}$, have very low probability of metastasis to the sentinel node, on average $5.2 \%$, and lymphatic mapping with SLNB is therefore not indicated. However, there are subgroups of patients in whom the procedure should be considered; the factor that predicts the presence of positive sentinel lymph node in melanomas with $<1 \mathrm{~mm}$ thickness with higher accuracy is tumor thickness; mean rate of metastases to the sentinel lymph node in patients with Breslow $<0.75 \mathrm{~mm}$ is $2.9 \%$, and in those between 0.75 and $1 \mathrm{~mm}$, it is $7.1 \%$.

Other factors that influence on and increase the possibility of metastatic sentinel lymph node in patients with thin melanomas include mitotic index $>1$ mitosis $/ \mathrm{mm}^{2}$, lymphovascular invasion, ulceration (infrequent in thin melanomas), lymphocyte tumor infiltration and regression. Factors that should be taken into account when the decision has to be made between lymphatic mapping with SLNB or surveillance; for some authors, the possibility of metastatic sentinel lymph node in the presence of at least one of the above-indicated factors is as high as $18 \%$.

Unlike intermediate melanomas, the prognosis is not poorer in patients with Breslow $<1 \mathrm{~mm}$ melanomas and metastatic sentinel lymph nodes.

In patients with sentinel lymph node metastasis, global consensus is to perform complementary lymphadenectomy of the area regardless of the type of metastasis, although currently we know that factors such as the number of metastatic sentinel lymph nodes, the presence of parenchymal and non-subcapsular metastases, ulceration and thickness of the primary tumor are directly proportionally associated with the possibility of metastasis to non-sentinel lymph nodes. The current value of complementary lymphadenectomy for metastatic sentinel node lies in that, on average, $21 \%$ of these patients will have lymph nodes with metastases at dissection, which allows to stratify them by recurrence risk; i.e., those who have metastases to non-sentinel lymph nodes have poorer survival and shorter disease-free interval than those with metastasis limited to the sentinel lymph node, and eventually they could be candidates for adjuvant therapies or clinical trials.

Recently published results of the MSLT-II trial, which compared complementary dissection versus follow-up in patients with metastatic sentinel lymph node, showed that although complementary dissection does not increase specific survival, it does offer higher regional control and prognostic information. ${ }^{6}$

In summary, lymphatic mapping with SLNB is the standard method for lymph node staging in patients with skin melanoma without palpable lymph node metastasis or ultrasound with Breslow depth $>1 \mathrm{~mm}$ and in those with $<1 \mathrm{~mm}$ and adverse prognostic factors; metastasis to the sentinel node is an indication for complementary lymphadenectomy to be performed, based on currently available evidence.

If the medical group treating the patient cannot perform the lymphatic mapping with SLNB, there are two attitudes that can be adopted: monitoring the lymph-carrying area or refer the patient to centers or groups that do it according to world standards (the latter attitude is preferred).

Lymphatic mapping with SLNB is not indicated in stage 0 melanomas or $<0.75 \mathrm{~mm}$ or thinner melanomas between 0.75 and $1 \mathrm{~mm}$, except if other risk factors coexist in the patient, although these are not yet properly established. The key to success is to stick to the recommended technique since the diagnostic process.

\section{References}

1. Gallegos JF ¿Qué es el ganglio centinela? Concepto y aplicaciones en oncología. Acta Med Grupo Angeles. 2005;3:91-98.

2. Morton DL, Wen DR, Wong JH, Economou JS, Cagle LA, Storm FJ, et al Technical details of intraoperative lymphatic zapping for early stage melanoma. Arch Surg. 1992;127:392-399.

3. Nieweg OE, Uren RF, Thompson JF. The history of sentinel node biopsy. Cancer J. 2015;21:3-6.

4. Morton DL, Thompson JF, Cochran AJ, Mozillo N, Nieweg OE, Roses DF, et al. Final trial report of sentinel-node biopsy vs nodal observation in melanoma. N Engl J Med. 2014;370:599-609.

5. Balch CM, Thompson JF, Gershenwald JE. Age as a predictor od sentinel node metastasis among patients with localized melanoma: an inverse correlation of melanoma mortality and incidence of sentinel node metastasis among young and old patients. Ann Surg Oncol. 2014;21:1075-1081.

6. Faries MB, Thompson JF, Cochran AJ, Andbacka RH, Mozillo N, Zager JS, et al. Completion dissection or observation for sentinel-node metastasis in melanoma. N Engl J Med. 2017;376:2211-2222. 